\title{
Lights, Camera, Action: Optimizing Virtual Video Visits to Provide High-Quality Care
}

\author{
Sarah Onorato, $B A^{\top}$, Ashwini Joshi, $B S E^{\top}{ }^{\infty}$, and Andrea Wershof Schwartz, MD, MPH $H^{1,2,3}$ \\ ${ }^{1}$ Harvard Medical School, Boston, MA, USA; ${ }^{2}$ New England Geriatrics Research Education and Clinical Center and Division of Geriatrics \& Palliative \\ Care, VA Boston Healthcare System, Boston, MA, USA; ${ }^{3}$ Brigham \& Women's Hospital, Boston, MA, USA.
}

J Gen Intern Med 36(6):1751-4

DOI: $10.1007 / \mathrm{s} 11606-020-06278-Z$

(c) Society of General Internal Medicine (This is a U.S. government work and not under copyright protection in the U.S.; foreign copyright protection may apply) 2020

\section{INTRODUCTION: TELEMEDICINE WITHOUT THE TRAINING}

The COVID-19 pandemic has fundamentally changed the way that patients interact with the United States health care system. As we seek to lower the risk of viral transmission and expand the capacity of health care facilities, live videoconferencing - the practice of conducting a synchronous, virtual, and interactive visit between a patient and clinician in place of an in-person clinical encounter ${ }^{1}$ - has been considered as a possible solution to these issues ${ }^{2}$ and has been rapidly rolled out in many health care settings. ${ }^{3}$ Acknowledging the value of virtual visits in the COVID-19 era, Medicare has committed to expanding reimbursement for telemedicine services. ${ }^{4}$

The definition of telehealth is broad, ${ }^{5}$ and includes both remote clinical encounters and other virtual methods of care delivery such as remote patient monitoring or electronic consults. Telemedicine refers specifically to clinical interactions directly involving communication between a clinician and patient through a variety of digital methods. ${ }^{6}$ Using definitions offered by the American Telemedicine Association, we will specifically focus on the type of telemedicine delivered through live videoconferencing in the outpatient setting, during which a clinician and patient engage in a synchronous, interactive clinical encounter via video, which we will refer to as "virtual video visits" or "virtual visits."

Prior to the pandemic, the use of telemedicine was increasing gradually, especially for primary care and mental health care. ${ }^{7}$ Though video visits are associated with high patient satisfaction $^{8,9}$ and may improve patient access and outcomes, ${ }^{9}$ many clinicians were not routinely offering video visits prior to COVID-19 and are likely conducting them for the first time during the pandemic. Moreover, formal training in conducting virtual visits is limited, even among those who may have some

Sarah Onorato and Ashwini Joshi contributed equally to this work.

Received July 1, 2020

Accepted September 28, 2020

Published online October 27, 2020 experience with telemedicine, ${ }^{10}$ as few medical schools offer telemedicine education. ${ }^{11}$ Given the unprecedented need for connecting with patients virtually during the COVID-19 era, it is important to consider how clinicians can translate their experience with in-person visits to virtual visits. These skills are increasingly important as telemedicine becomes an integrated part of our routine care delivery system, both as a primary modality for outpatient visits and for triaging patients who may require in-person evaluation.

Much like in-person visits, virtual visits include distinct components that can be optimized through the use of specific best practices. We present a chronological framework for conducting telemedicine visits that addresses strategies for successfully navigating each of the three phases of the visit: setting up the visit, obtaining the history and conducting the physical examination, and presenting the assessment and plan. The phrase "lights, camera, action," describes each of these three phases, respectively, and can be used to help organize an approach to the virtual visit.

\section{LIGHTS: SETTING UP A SYNCHRONOUS VIRTUAL VIDEO VISIT}

The setup of a virtual visit, while often underappreciated, is critical to the success of the encounter. A virtual setup that minimizes distractions and conveys professionalism can put the patient at ease and promote a therapeutic alliance. Conversely, an encounter without adequate setup or riddled with technological difficulties can detract from clinical time, erode patient trust, and adversely affect patient experience. We will consider particular strategies that clinicians can use to prepare themselves and their patients for a virtual encounter. These steps can be further described as addressing the who, what, when, where, why, and how of the virtual encounter.

In thinking about who should be included in the virtual visit, clinicians should ensure that all relevant team members are present and have a clearly defined role, including the patient's caregiver, an interpreter if required, interprofessional team members, and trainees. A medical assistant should provide clear instructions on how to access the visit and how the patient will be contacted. It is important to recognize that many patients may have never participated in a virtual video visit and may be apprehensive about using this modality. Clinicians can orient 
patients to the virtual visit in the same way they might ordinarily introduce patients to a new clinic location, in addition to obtaining informed consent and assuring patient confidentiality. During the visit, clinicians can enhance the patient's experience by retaining the same skills used in clinic to make eye contact, communicate warmth, and demonstrate attention (Table 1).

The next step is the what of the visit, which focuses on optimizing the video experience for patients and setting up the necessary items beforehand. These steps include optimizing internet, camera, and microphone quality; ensuring adequate lighting such that patients can see facial expressions well; maintaining a neutral background; and wearing solid-colored clothing to minimize distractions during video transmission. In addition, all materials and equipment should be placed within reach before the visit so that clinicians will not need to move off screen during the encounter. Clinicians should establish a backup plan in case connectivity problems arise during the visit, such as an additional source of internet connection or a phone line if video fails.

In terms of when these visits occur, it is important first to identify which patients and chief concerns are appropriate for virtual visits and which are more appropriate for in-person evaluation. For example, when a patient might require an inperson examination to guide clinical decision-making, when additional testing may be required, or when an office-based procedure is anticipated, clinicians should strongly consider scheduling patients for evaluation in an in-person clinical setting. The virtual visit can also be used as a triaging tool to identify patients who would benefit from additional in-person evaluation. A protocol should be in place for bringing patients in for in-person evaluation or referring them to emergency services as appropriate.

When considering where these visits occur, it is important to pay attention to both the physical and virtual space. Clinicians should confirm the patient's location and contact information in case of an emergency. Furthermore, clinicians should scan and lock the virtual room to ensure patient security and confidentiality. It is important to confirm that patients are in a private space or that their confidentiality is protected in the event that they are unable to find a private space in which to engage in the virtual visit.

In considering how virtual visits are best conducted, it is crucial for clinicians to focus on the patient, rather than the virtual visit platform. Clinicians should be mindful of looking directly at the camera-which should be centered and well positioned - to communicate eye contact. When it is necessary to look at the keyboard, notes, or a computer monitor or to interact with the electronic health record during the visit, clinicians can communicate their actions to the patient to reduce the likelihood of appearing distracted. ${ }^{12}$

Given the potential challenges associated with engaging in video visits, it is helpful to recall why these visits are valuable for both patients and clinicians. Benefits include increasing specialty care access, minimizing infection exposure risk for clinicians and patients, and providing a convenient, efficient, cost-effective way to connect with patients and other care team members. ${ }^{2}$ However, it is also equally critical to acknowledge the limitations of this modality and to recognize that video visits may not be appropriate for all patients, such as those who require in-person evaluation or prefer additional privacy. Clinicians should also be mindful of potential socioeconomic

Table 1 Lights, Camera, Action: Translating Face-to-Face Clinician Actions into Virtual Strategies

\begin{tabular}{|c|c|}
\hline In-person & Virtual visit \\
\hline \multicolumn{2}{|l|}{ Lights: visit setup } \\
\hline Patient schedules an appointment & $\begin{array}{l}\text { Patient schedules a virtual visit and a team member provides instructions on how } \\
\text { patient will be contacted and how to access visit }\end{array}$ \\
\hline $\begin{array}{l}\text { If needed, clinician calls interpreter or arranges for in-person } \\
\text { interpreter }\end{array}$ & All team members, including interpreters, are present virtually \\
\hline $\begin{array}{l}\text { Ensure clean exam room with necessary medical supplies and } \\
\text { lighting }\end{array}$ & Optimize internet, microphone, and camera quality, with backup connectivity plan \\
\hline $\begin{array}{l}\text { Identify patients as having a routine vs. urgent concern and have } \\
\text { a triage plan in case of emergencies }\end{array}$ & Confirm patient location and contact information in case of emergency \\
\hline $\begin{array}{l}\text { Takes place in an examination room with door/curtain for } \\
\text { privacy }\end{array}$ & Ensure clinician and patient are each in private location and virtual room is secure \\
\hline \multicolumn{2}{|l|}{ Camera: history and physical exam } \\
\hline Clinician introduces self to patient & Orient patient to video visit \\
\hline Make eye contact & Look directly at the camera, not solely at the patient's picture on the screen \\
\hline Speak slowly and clearly & Ensure microphone quality; consider using a headset for patient and/or clinician \\
\hline Establish clear goals for the visit & $\begin{array}{l}\text { Establish clear goals for the virtual visit, including determination of if an in-person } \\
\text { follow up visit is necessary }\end{array}$ \\
\hline $\begin{array}{l}\text { Include caregivers and family members in exam room when } \\
\text { appropriate }\end{array}$ & $\begin{array}{l}\text { Include caregivers and family members virtually, when appropriate, by arranging } \\
\text { for them to join even if not co-located with patient }\end{array}$ \\
\hline "Brown bag" medication review & Patient holds medication bottles, pillbox, or list up to screen \\
\hline $\begin{array}{l}\text { Perform observation, percussion, palpation, auscultation, and } \\
\text { focused maneuvers of patient }\end{array}$ & $\begin{array}{l}\text { Observe patient and their environment, engage patient or caregivers to assist with } \\
\text { exam maneuvers or assessments as appropriate }\end{array}$ \\
\hline \multicolumn{2}{|l|}{ Action: assessment and plan } \\
\hline Education, instructions, paperwork printed for patient & Education, instructions, paperwork mailed, faxed, or sent via secure message \\
\hline $\begin{array}{l}\text { Clinician asks the patient if they have any questions before the } \\
\text { clinician leaves the room }\end{array}$ & Clinician asks the patient if they have any questions before the patient ends the call \\
\hline
\end{tabular}


barriers to access to video visits and work within their health care system to eliminate disparities. ${ }^{13}$

\section{CAMERA: OBTAINING THE HISTORY AND CONDUCTING A PHYSICAL EXAM}

History-taking skills are readily transferred to, and even enhanced by, the virtual setting. Virtual video visits may allow the clinician to observe the patient in their living environment, helping them to better understand the context in which patients live and manage their medical conditions. For example, when reviewing a patient's medications, clinicians can ask patients to hold up their prescription bottles to the screen and demonstrate how they organize them. Clinicians should ask the same focused questions used during an in-person encounter, using their individual area of expertise as guidance.

The physical exam remains an important part of virtual visits and can help determine whether a patient needs to be evaluated in person. Although observation is already known to be important during in-person clinical encounters, ${ }^{14}$ this skill is paramount for virtual examinations. Clinicians can observe a patient's environment, grooming, general appearance, mental status, effort of breathing, and musculoskeletal range of motion via video. Details such as dry mucous membranes or poor dentition may be appreciated, and a limited neurologic or dermatologic exam may be accomplished. ${ }^{15,16}$ Observation is perhaps most important in clinical decision-making regarding triaging patients for further in-person evaluation; an illappearing patient must be identified over video using observation skills clinicians typically rely on to determine how sick or well a patient is based on their general appearance.

Vital sign measurement and other exam maneuvers can also be performed virtually. For instance, if patients have a blood pressure cuff at home, the clinician can observe their technique and obtain blood pressure readings. Focused exam maneuvers can also be conducted virtually, with the help of the patient or a caregiver if needed. For example, in an older patient, a safely executed chair stand can examine mobility, while visual inspection of a patient's toenails may help the clinician understand capability for self-care. ${ }^{17}$ In addition, certain assessments such as the Montreal Cognitive Assessment (MOCA) and assessment for Parkinson's disease have been studied and validated for use in virtual settings. ${ }^{18,19}$ More research is urgently needed to validate additional methods of assessing patients via virtual video visit.

\section{ACTION: PRESENTING AN ASSESSMENT AND PLAN}

Like a face-to-face visit, a virtual visit concludes with an assessment and plan. There are many similarities between concluding in-person visits and virtual visits, though clinicians must plan ahead regarding the best way to transmit information that would normally be handed to a patient at the conclusion of an in-person encounter. Clinicians can provide verbal education and share written instructions and educational materials via secure electronic message with patients and caregivers, or by mail or fax if necessary. In addition, clinicians should continue to electronically complete important forms that would typically be filled out with the patient in person, such as portable orders for life-sustaining treatment (POLST) forms for advance care planning, although the logistics of obtaining signatures should be considered in advance in states that have not waived this requirement. ${ }^{20}$

Special consideration should be given to plans in the event that urgent or emergent issues arise during the course of the virtual video visit. If clinicians are concerned about a patient, they can offer instructions for the safest way for the patient to present for in-person evaluation, including calling emergency services on the patient's behalf if necessary. Clinicians will ideally have collected and reviewed these contingencies with the patient as a part of the setup phase of the visit.

It is important to allow the patient to end the virtual visit once education has been provided, a plan for action items has been communicated, and all questions have been answered. Making sure that the patient, rather than the clinician, ends the call, avoids the "doorknob phenomenon" 21 and creates space for the patient to express any final thoughts before definitively ending the visit by pressing a button, in contrast to the several minutes that can elapse between a clinician leaving an examination room and the patient physically leaving the clinic. Once the visit has ended, any clinicians or trainees involved in the visit can reflect on the encounter, debrief if necessary, and review next steps.

Clinicians can translate existing skills into virtual strategies that prioritize patient satisfaction, experience, and outcomes. Virtual visits are critical during the current pandemic, as they allow clinicians to continue to provide routine care to patients for whom it is not safe, advisable, or feasible to be seen in person. The rapid implementation of virtual video visits in the era of COVID-19 provides an opportunity for this efficient model of care to become an increasingly integrated part of our health care system with the potential to improve the accessibility, timeliness, and quality of care provided to patients.

Acknowledgments: We thank Kelly Doherty for assistance with the literature review. AS would like to thank her colleagues, trainees, and patients in the VA Boston TeleGeriatrics clinic. AS previously presented this material orally as part of a seminar for VA and Harvard Medical School Faculty and for the Institute for Healthcare Improvement's Age Friendly Health Systems Initiative, which was cited on their blog. ${ }^{22}$ Some of this material is the result of work supported with resources and the use of facilities at the Veterans Health Administration Boston Medical Center and the New England Geriatric Research Education and Clinical Center. The views expressed in this article are those of the authors and do not necessarily reflect the position or policy of the Department of Veterans Affairs or the United States government.

Corresponding Author: Ashwini Joshi, BSE; Harvard Medical School, Boston, MA, USA (e-mail: ashwini_joshi@hms.harvard.edu).

Compliance with Ethical Standards: 
Conflict of Interest: SO, AJ, and AWS have no conflicts of interest. This research did not receive any specific grant from funding agencies in the public, commercial, or not-for-profit sectors.

\section{REFERENCES}

1. American Telemedicine Association. Telehealth Basics [Internet]. 2020 [cited 2020 Aug 9];Available from: https://www.americantelemed.org/ resource/why-telemedicine/

2. Hollander JE, Carr BG. Virtually Perfect? Telemedicine for Covid-19. N Engl J Med 2020;382(18):1679-81.

3. Peden CJ, Mohan S, Pagán V. Telemedicine and COVID-19: an Observational Study of Rapid Scale Up in a US Academic Medical System. J Gen Intern Med [Internet] 2020 [cited 2020 Jun 27];Available from: http:// link.springer.com/10.1007/s11606-020-05917-9

4. Centers for Medicare \& Medicaid Services. Medicare Telehealth Healthcare Provider Fact Sheet [Internet]. 2020 [cited 2020 Jun 27];Available from: https://www.cms.gov/newsroom/fact-sheets/ medicare-telemedicine-health-care-provider-fact-sheet

5. Tuckson RV, Edmunds M, Hodgkins ML. Telehealth. N Engl J Med 2017;377(16):1585-92.

6. Centers for Disease Control and Prevention. Telehealth and Telemedicine: A Research Anthology of Law and Policy Resources [Internet]. 2019 [cited 2020 Aug 9];Available from: https://www.cdc.gov/phlp/publications/ topic/anthologies/anthologies-telehealth.html

7. Barnett ML, Ray KN, Souza J, Mehrotra A. Trends in Telemedicine Use in a Large Commercially Insured Population, 2005-2017. JAMA 2018;320(20):2147.

8. Dorsey ER, Topol EJ. State of Telehealth. N Engl J Med 2016;375(2):154-61.

9. U.S. Department of Veterans Affairs. VA Telehealth Services Factsheet [Internet]. [cited 2020 Jun 27]; Available from: https://connectedcare.va. gov/sites/default/files/OT_va-telehealth-factsheet-2019-01.pdf

10. Edirippulige $\mathbf{S}$, Armfield $\mathbf{N}$. Education and training to support the use of clinical telehealth: A review of the literature. $J$ Telemed Telecare 2017;23(2):273-82.

11. Waseh S, Dicker AP. Telemedicine Training in Undergraduate Medical Education: Mixed-Methods Review. JMIR Med Educ 2019;5(1):e12515.
12. American Medical Association. Telehealth Visit Etiquette Checklist [Internet]. [cited 2020 Jun 20]; Available from: https://www.ama-assn. $\mathrm{org} / \mathrm{system} /$ files/2020-04/telehealth-appendix-g4-telehealth-visit-etiquette-checklist.pdf

13. Nouri SS, Avila-Garcia P, Cemballi AG, Sarkar U, Aguilera A, Lyles CR. Assessing Mobile Phone Digital Literacy and Engagement in UserCentered Design in a Diverse, Safety-Net Population: Mixed Methods Study. JMIR MHealth UHealth 2019;7(8):e14250.

14. Naghshineh S, Hafler JP, Miller AR, et al. Formal Art Observation Training Improves Medical Students' Visual Diagnostic Skills. J Gen Intern Med 2008;23(7):991-7.

15. Boyers LN, Schultz A, Baceviciene R, et al. Teledermatology as an Educational Tool for Teaching Dermatology to Residents and Medical Students. Telemed E-Health 2015;21(4):312-4.

16. Al Hussona M, Maher M, Chan D, et al. The Virtual Neurologic Exam: Instructional Videos and Guidance for the COVID-19 Era. Can J Neurol Sci 2020;1-6.

17. Schwartz AW. What Van Halen Can Teach Us About the Care of Older Patients. JAMA Intern Med 2017;177(3):309.

18. Iiboshi $\mathbf{K}$, Yoshida $\mathbf{K}$, Yamaoka $\mathbf{Y}$, et al. A Validation Study of the Remotely Administered Montreal Cognitive Assessment Tool in the Elderly Japanese Population. Telemed E-Health 2020;26(7):920-8.

19. Abdolahi A, Scoglio N, Killoran A, Dorsey ER, Biglan KM. Potential reliability and validity of a modified version of the Unified Parkinson's Disease Rating Scale that could be administered remotely. Parkinsonism Relat Disord 2013;19(2):218-21.

20. Calton BA, Rabow MW, Branagan L, et al. Top Ten Tips Palliative Care Clinicians Should Know About Telepalliative Care. J Palliat Med 2019;22(8):981-5.

21. Faden J, Gorton G. The Doorknob Phenomenon in Clinical Practice. Am Fam Physician 2018;98(1):52-3.

22. IHI Multimedia Team. Tips You May Not Know to Improve Telehealth for Patients and Providers [Internet]. 2020 [cited 2020 Aug 15];Available from: http://www.ihi.org/communities/blogs/tips-you-may-not-knowto-improve-telehealth-for-patients-and-providers

Publisher's Note: Springer Nature remains neutral with regard to jurisdictional claims in published maps and institutional affiliations. 